\title{
Accretion disks around massive stars: Hydrodynamic structure, stability and dust sublimation
}

\author{
Bhargav Vaidya ${ }^{a}$, Christian Fendt and Henrik Beuther \\ Max Planck Institute for Astronomy, Königstuhl 17, D-69117 Heidelberg, Germany \\ vaidya@mia.de, fendt@mpia.de, beuther@mpia.de
}

\begin{abstract}
We investigate the structure of accretion disks around massive protostar applying steady state models of thin disks. The thin disk equations are solved with proper opacities for dust and gas taking into account the huge temperature variation along the disk. We explore a wide parameter range concerning stellar mass, accretion rate, and viscosity parameter $\alpha$. The most essential finding is a very high temperature of the inner disk. For e.g. a $10 \mathrm{M}_{\odot}$ protostar and an accretion rate of $\sim 10^{-4} \mathrm{M}_{\odot} \mathrm{yr}^{-1}$, the disk midplane temperature may reach almost $10^{5} \mathrm{~K}$. The disk luminosity in this case is about $10^{4} \mathrm{~L}_{\odot}$ and, thus, potentially higher than that of a massive protostar. We motivate our disk model with similarly hot disks around compact stars. We calculate a dust sublimation radius by turbulent disk self-heating of more than $10 \mathrm{AU}$, a radius, which is 3 times larger than caused by stellar irradiation. We discuss implications of this result on the flashlight effect and the consequences for the radiation pressure of the central star. In difference to disks around low mass protostars our models suggest rather high values for the disk turbulence parameter $\alpha \leq 1$. However, disk stability to fragmentation due to thermal effects and gravitational instability would require a lower $\alpha$ value. For $\alpha=0.1$ we find stable disks out to $80 \mathrm{AU}$. Essentially, our model allows to compare the outer disk to some of the observed massive protostellar disk sources, and from that, extrapolate on the disk structure close to the star which is yet impossible to observe.
\end{abstract}

Subject headings: accretion, accretion disks - hydrodynamics - methods : analytical - stars: formation turbulence

\section{Introduction}

Massive stars play a vital role in order to understand the dynamical evolution of clusters in which they are the major source of heavy elements and UV radiation. During their short life time, they impact their surrounding by a number of physical processes such as jet like outflows, strong winds, photo evaporation, expanding HII regions and eventually supernova explosions (Zinnecker \& Yorke 2007; Beuther 2007). Collimated molecular outflows and jets from young massive protostars have been observed

${ }^{a}$ Member of the International Max Planck Research School for Astronomy and Cosmic Physics at University of Heidelberg, IMPRS-HD
(Beuther et al. 2002; Beuther \& Shepherd 2005), although the launching mechanism for outflows have been mainly investigated for low mass protostars so far (see e.g. Casse \& Keppens 2002; Pudritz et al. 2007; Fendt 2009).

Understanding massive star formation has been a very active field of research for both the observers and the theorists. The basic differences of high and the low mass star formation process are that of timescales, energy scales, mass flow rates and high luminosity of the central star. Low mass stars have well defined phases in their formation process and they only start burning hydrogen after accretion of all matter is done (Stahler \& Palla 2005). In contrast, massive stars have very short Kelvin Helmholtz time of $\sim 10^{4}-10^{5} \mathrm{yr}$, and thus 
start burning hydrogen even when they are still accreting. One of the problems in the formation of massive stars is that, at some point in time, the strong radiation pressure from the luminous central star may exceed the Eddington limit in spherical symmetry and does not allow the matter to accrete anymore. This seems to limit the mass of the star to be formed via the spherical accretion to about $20 \mathrm{M}_{\odot}$ (Wolfire \& Cassinelli 1987).

However, disk accretion may add dynamical pressure to the accreting matter which may help overcoming the stellar radiation pressure. Thus, the star formation scenario for low and high mass stars could in principle be the same (e.g. Keto 2007), suggesting that high mass star formation as a scaled up version of the low mass star formation process. With our paper we aim to construct a global model (length scales of $\sim 0.1 \mathrm{AU}$ to $100 \mathrm{AU}$ ) of the accretion disk around massive young stars. By fitting the outer disk structure to the observations, this will allow us to investigate, in follow up projects, the feasibility of certain physical processes in inner disk which could be essential for the pre-stellar evolution as angular momentum transport or processes responsible for launching of outflows.

Our paper relies on one essential assumption - that is the disk accretion rate. Both estimates following the observed outflow rates and the time scale of mass aggregation suggest accretion rates in the range of $10^{-3}-10^{-4} \mathrm{M}_{\odot} \mathrm{yr}^{-1}$ (Beuther et al. 2002; Zhang 2005; Grave \& Kumar 2009). However, one of the aims of our study is to understand whether such values are compatible with theoretical accretion disk models.

In the following section of this paper, we discuss about the modelling of the disk structure using the standard disk equations, in $\S 3$ we describe the disk opacity model which has contribution from both, dust and gas and in $\S 4$ we present our results and give estimates of the sublimation radius and then in $\S 5$ discuss about the stability of the disk and how the viscosity parameter $\alpha$ has an effect on it. Also in $\S 6$ we compare our results with the recent observations of disks in massive young protostars. Finally, in $\S 7$ we present our conclusions from this simple approach to study disks around massive stars.

\section{Accretion Disk Model}

In order to study the global disk structure around massive stars, we apply the thin disk model (Shakura \& Syunvaev 1973; Morfill \& Wood 1989; Ruden \& Pollack 1991; Frank et al. 1992; Stepinski et al. 1993; Bell \& Lin 1994) with appropriate modifications. The essential part of our model is to take into account the proper gas and dust opacities for a huge temperature regime as indicated for high accretion rates on to massive stars. The original standard disk model by Shakura \& Svunvaev (1973) relates to solving the stationary state hydrodynamic equations for the thin disk assuming certain opacity laws for different regions in the disk. The disk is classified to have three regions $(\mathrm{A}, \mathrm{B}$, and $\mathrm{C})$ depending upon the opacity and the pressure. The region $\mathrm{C}$ is the outermost region where the gas pressure is much greater than the radiation pressure in the disk and free-free opacity dominates in this region, whereas region $\mathrm{B}$ is the one in which gas pressure is still greater but the Thomson scattering is now dominating over Kramer's opacity. The region A is the one which is closest to the central object and here the radiation pressure in the disk is much greater than the gas pressure. The thin disk model was extended to include dust opacities and has been applied to disks around low mass stars and solar nebula. (Ruden \& Pollack 1991; Stepinski et al. 1993; Stepinski 1998; Del Popolo \& Ekși 2002). The application of such a thin disk model to massive stars is a simple approach to understand the dynamics of the disk especially in the inner most region which can not be resolved with the help of present day telescopes. We look for steady state solution of the thin disk model using the hydrodynamic equations of the thin disk (e.g. Frank et al. 1992). The vertically averaged surface density $\Sigma$ is related to the mass density $\rho$ and the scale height $\mathrm{H}$.

$$
\Sigma=2 H \rho
$$

The scale height is estimated using the approximation for thin disk

$$
H=\sqrt{2}\left(\frac{c_{s}}{\Omega}\right)
$$

where $\Omega$ is the Keplerian angular velocity and $c_{s}$, the sound speed is defined in terms of temperature and other physical constants like Boltzmann constant $k$, proton mass $m_{p}$ and the mean molecular 
weight $\mu$.

$$
c_{s}^{2}=\left(\frac{k}{\mu m_{p}}\right) T
$$

The energy balance equation which implies that the amount of energy radiated from the disk is equal to the amount of energy produced by viscous heating.

$$
\left(\frac{16 \sigma}{3 \Sigma \kappa}\right) T^{4}=\left(\frac{9}{4}\right) \nu \Sigma \Omega^{2}
$$

The viscosity $\nu$ is defined by introducing the parameter $\alpha$.

$$
\nu=\alpha c_{s} H
$$

The Rosseland mean opacity $\kappa$ is related to the mass density and the temperature in form of power law with $\kappa_{0}$ as constant.

$$
\kappa=\kappa_{0} \rho^{m} T^{n}
$$

The condition for the conservation of angular momentum in steady state can also be derived.

$$
\Sigma \nu=\frac{\dot{M}}{3 \pi}\left[1-\left(\frac{R_{*}}{r}\right)^{\frac{1}{2}}\right]
$$

The dimensionless viscosity factor $\alpha$ is introduced basically to parameterize viscosity by assuming that is provided by turbulent motions present in the disk. The physical significance of equation (5) is that the largest size of turbulent eddy that can be sustained in the disk is of size equal to the disk scale height and maximum speed of the turbulent motion is that of sound speed, this implies values of $\alpha \lesssim 1$. In order to solve the above equations consistently, one needs the form of opacity. For the opacity of the form of a power law given by equation (6), one gets power law solutions of all the dynamical quantities in the disk. For instance, in case of the form of Kramer opacity law $\left(\kappa=6.6 \times 10^{-22} \rho^{1} T^{-\frac{7}{2}}\right)$ used by Shakura \& Syunyaev (1973), the central temperature in the disk around a typical massive star in the region where $P_{\text {gas }}>P_{\text {rad }}$ and $\sigma_{\mathrm{ff}}>\sigma_{\mathrm{t}}$ is

$$
\begin{aligned}
T_{\mathrm{c}}(r)=1.74 \times 10^{4} \mathrm{~K}(\alpha)^{-1 / 5}\left(\frac{\dot{M}}{10^{-4} \mathrm{M}_{\odot} \mathrm{yr}^{-1}}\right)^{3 / 10} \\
\left(\frac{M}{10 \mathrm{M}_{\odot}}\right)^{1 / 4}\left(\frac{r}{10 \mathrm{R}_{\star}}\right)^{-3 / 4}\left(\frac{\mathrm{R}_{\star}}{6 \mathrm{R}_{\odot}}\right)^{-3 / 4}(8)
\end{aligned}
$$

where $\sigma_{\mathrm{ff}}$ is the free free opacity and $\sigma_{\mathrm{t}}$ is the opacity due to electron scattering.

\section{Disk opacities}

The work done by Shakura \& Syunyaev (1973) was mainly focused on hot accretion disks around black holes, the contribution to the opacity in their case was mainly from the electron scattering or free-free emission depending upon the optical depth in the disk. However in disks around massive young stars, contribution to opacity also comes from dust present beyond the sublimation radius, many molecular and atomic lines and other scattering processes. Since massive stars have large radiation fields that heavily affect the huge amount of dust present in the envelope and also the gas that is present in the inner part, one has to take into account a proper contribution of opacities from dust and gas to have a consistent accretion disk model.

In our model the dust opacity does not have any explicit dependence on frequency, so the effective value of the opacity

$$
\kappa_{\mathrm{eff}}=\frac{\int \kappa_{\nu} F_{\nu} d \nu}{\int F_{\nu} d \nu}
$$

simplifies to $\kappa_{\text {eff }}=\kappa_{\nu}$

In our work, we follow that the matter is accreted via a disk investigating the possible location of the dust sublimation radius. We expect that the active disks will be dominating in destroying the dust in the disk at a much further radius then it would have been destroyed by the heating from star. This would result in lowering the radiation pressure on the dust and allowing the infall of matter on the central star.

Apart from some metal silicates all the dust grains that are present sublimates at around $1500 \mathrm{~K}$. Since at this temperature, the gas and the dust opacities vary substantially, it is essential for calculating radiation pressure to consider the proper opacity.

\subsection{Dust opacities}

There are many factors one has to take into account for getting a consistent model for dust opacity such as the size of the grains, distribution of grains in the disk and the coagulation of dust grains. Also the temperature of the disk varies over a large range and this would clearly affect the composition of the dust and alter the gas to 
dust ratio typically $\sim 100$ in the disk. This radial variation in the gas to dust ratio will not only modify the gas pressure along the disk but also the other dynamical disk quantities. The usual assumption in many of the dust models is that the dust and gas are well coupled and also the gas to dust ratio is taken to be 100 . In general, opacity has a typical dependence on temperature and density. (e.g. Morfill \& Wood 1989; Ruden \& Pollack 1991; Bell \& Lin 1994; Ossenkopf \& Henning 1994; Helling et al. 2000; Semenov et al. 2003).

In the regime which is dominated by dust $\left(r \gg R_{*}\right)$, the opacity depends very weakly on density. In the present work we apply for the dust dominated region the model proposed by Ruden \& Pollack (1991) in which the Rosseland mean opacities have been given in terms of the power laws of the form given in equation (6), in different regimes that have been distinguished on the basis of temperature and mass density (see Table 11). Using this power law form of opacity, equations (11) - (6) can be reduced to a form representing the viscosity as a function depending on surface density and the radial distance as

$$
\nu=C \Sigma^{p} r^{q}
$$

This form of viscosity can be then substituted in equation (77) so that all the dynamical quantities namely the surface density, mass density, central temperature, scale height of the disk can be expressed as power laws of mass, accretion rate, radial distance and the viscosity parameter $\alpha$. For the parameters of a typical massive star these quantities $(\mathrm{Q})$ can be written

$$
\begin{array}{r}
Q=C_{i}\left(\frac{M_{*}}{\mathrm{M}_{\odot}}\right)^{\gamma_{i}}\left(\frac{r}{R_{i}}\right)^{\epsilon_{i}} \alpha^{\delta_{i}} \\
\left(\frac{\dot{M}}{10^{-3} \mathrm{M}_{\odot} \mathrm{yr}^{-1}}\right)^{\beta_{i}}
\end{array}
$$

e.g. (Ruden \& Pollack 1991; Stepinski et al. 1993; Del Popolo \& Eksi 2002). The index $i$ denotes the opacity regime as given in the Table $1 . \quad(i=$ $1,2 \ldots 6), C_{i}$ are constants and $R_{i}$ is chosen as a typical radius for that regime. The dust model taken into account does not consider explicit wavelength dependence of opacity.

\subsection{Gas opacities}

The opacity of gas and dust have been estimated consistently. These opacities are usually listed in form of a table in two parameters space, temperature $(\mathrm{T})$ and parameter $(\mathrm{R})$ which is dependent on density in a following manner

$$
R=\frac{\rho}{\left(T_{6}\right)^{3}}
$$

(Iglesias \& Rogers 1996; Ferguson et al. 2005) where $\rho$ is the density and $T_{6}$ is the temperature normalized to $10^{6} \mathrm{~K}$. These opacities also show some dependence on metallicity as well.

In a regime where dust begins to sublimate and molecules start to form, there is a sharp decrease in the opacity. In this regime, dependence of opacity on density is strong, unlike the opacity due to dust, and in general it is very difficult to get the true value of the opacity in this region so usually linear interpolation is used (Semenov et al. 2003). Due to steep turn over of the opacity gradient with the temperature, numerical difficulties arise when the dynamical quantities are estimated for this region. In the present work, we take into account the opacity tabl $\Theta^{7}$ by Ferguson et al. (2005), which has the temperature range of our relevance $\left(500 \mathrm{~K}-10^{4.5} \mathrm{~K}\right)$. For the present purpose we choose a table with hydrogen fraction $\mathrm{X}$ $=0.94$ and metallicity $\mathrm{Z}=0.06$. In fact, we also in the inner most region obtain higher temperatures and so we apply in continuation the OPAL opacity table $\mathrm{A}$ for higher temperatures $\left(\geq 10^{4.15} \mathrm{~K}\right)$ (Iglesias \& Rogers 1996). We create equispace grid of two parameters $\log (\mathrm{T})$ and $\log (\mathrm{R})$ as defined above and put the values from the table in the grid and fit a $2 \mathrm{D}$ cubic spline on it to get the interpolated values of opacity for any given temperature and density, but as the regime where the gas and dust coexists is difficult to model numerically as the opacity do not converge to a unique value, we make a linear approximation as zeroth order approach and compare with the analytical models present. (e.g. Ruden \& Pollack 1991; Bell \& Lin 1994).

\footnotetext{
${ }^{\dagger}$ http://webs.wichita.edu/physics/opacity

$\ddagger$ http://www-phys.llnl.gov/Research/OPAL/opal.html
} 
Table 1: Different regimes of the opacity taken from Ruden \& Pollack (1991) with the conditions for the change over and description of the main components of dust in each regime

\begin{tabular}{cccc}
\hline \hline Regime & $\kappa_{i}$ & Condition & Description \\
\hline 1 & $\left(2 \times 10^{-4}\right) \rho^{0} T^{2}$ & $T \leq 150 \mathrm{~K}$ & dominated by Water and Ice grains \\
2 & $\left(1.15 \times 10^{18}\right) \rho^{0} T^{-8}$ & $T \leq 180 \mathrm{~K}$ & Sublimation of Ice grains \\
3 & $\left(2.13 \times 10^{-2}\right) \rho^{0} T^{0.75}$ & $T \leq 1380 \mathrm{~K}\left(\frac{\rho}{10^{-8}}\right)^{1 / 50}$ & mainly consists of Iron and Silicate grains \\
4 & $\left(1.57 \times 10^{60}\right) \rho^{3 / 8} T^{-18}$ & $T \leq 1890 \mathrm{~K}\left(\frac{\rho}{10^{-8}}\right)^{1 / 48}$ & Sublimation of refractory grains \\
5 & $\left(1.6 \times 10^{-2}\right) \rho^{0} T^{0}$ & $T \leq 2620 \mathrm{~K}\left(\frac{\rho}{10^{-8}}\right)^{2 / 27}$ & Molecular and atomic lines contribute \\
6 & $\left(2 \times 10^{34}\right) \rho^{2 / 3} T^{-9}$ & $T \leq 3200 \mathrm{~K}$ & Molecular and atomic lines contribute \\
\hline \hline
\end{tabular}

\section{Results and Discussion}

In the following we present results of applying above standard models of disk accretion and opacities to massive star formation. We first discuss the outer dust dominated disk and then the inner gaseous disk.

\section{1. $\quad$ Outer disk structure : Radial Profiles of Dynamical Quantities}

The main contribution of opacity in the outer disk is from dust. The numerical values for the dynamical quantities expressed as given by equation (11) in different opacity regimes are listed in Table 2. The radial profile of the disk midplane temperature, surface density, mass density and scale height for a high mass star is shown in Fig. 1 for a $10 \mathrm{M}_{\odot}$ star with accretion rate of the order of $4.2 \times 10^{-4} \mathrm{M}_{\odot} \mathrm{yr}^{-1}$ and the a viscosity parameter $\alpha=1$. With these parameters, the central temperature reaches $1500 \mathrm{~K}$ around $12 \mathrm{AU}$ due to viscous heating, which causes the dust to sublimate. Figure 1 shows two curves for each quantity. The solid curve is obtained by implementing the opacity power laws as given by Ruden \& Pollack (1991), whereas the dashed line is obtained by using the opacities by Stepinski et al. (1993) and extrapolating it to higher mass stars.

There are few kinks seen in the plot, which are due to the fact that the dust opacity model used comprises of different regimes and in each of these regimes have a different form of power law (see Table 1). These regimes are connected using the standard procedure (Lin \& Papaloizou 1985) of equating the opacity in two consecutive regimes, $\kappa_{i}=\kappa_{i+1}$.
Since formation of massive stars involves large accretion rates the surface density and the mass density in the disk is higher than the lower mass counterparts, as more matter will be injected in the inner region. We find the scale height ratio obtained from the present model is approximately constant in the outer disk, with a radial dependence $\propto r^{0.28}$ and consistent with the thin disk approximation.

A least square linear fit of dynamical quantities gives for the temperature profile a power for the radial distance as -0.45 and for density as 2.35. The surface density has a rather flat profile as the best fit gives the dependence of the form $\propto r^{-1.1}$. These profiles are similar to those obtained by Ruden \& Pollack (1991) but here applied for a high mass star with high accretion rate.

The parameter values discussed above were chosen i) to have most part of the inner $100 \mathrm{AU}$ disk to be gravitationally stable and also ii) to ensure that the dust in the disk sublimates at a distance substantially further than caused by sublimation from stellar irradiation. (see below)

\subsection{Sublimation of dust}

Most of the dust grains sublimate when the temperature in the disk reaches the critical value of $1500 \mathrm{~K}$. The radius of the disk at which this value of critical temperature is reached is the dust sublimation radius. The disk can be heated by various processes such as viscous heating, stellar irradiation, convection, cosmic rays (D'Alessio et al. 1998). However, in the present work we estimate the dust sublimation radius via two main processes namely viscous heating and stellar irradiation (see 
Table 2: The various dynamical quantities for the massive star using the interpolated Rosseland mean dust opacity model given by Ruden \& Pollack (1991).(see equation (11) and Table 1)

\begin{tabular}{|c|c|c|c|c|}
\hline & \multicolumn{4}{|c|}{ Regime $1 R_{1}=400 A U$} \\
\hline $\mathrm{Q}$ & $T[\mathrm{~K}]$ & Height $[\mathrm{cm}]$ & $\rho\left[\mathrm{gcm}^{-3}\right]$ & $\Sigma\left[\mathrm{gcm}^{-2}\right]$ \\
\hline$C_{i}$ & 126.3 & $9.9 \times 10^{14}$ & $1.3 \times 10^{-13}$ & 258.8 \\
\hline \multicolumn{5}{|c|}{ Regime $2 R_{2}=250 A U$} \\
\hline $\mathrm{Q}$ & $T[\mathrm{~K}]$ & Height $[\mathrm{cm}]$ & $\rho\left[\mathrm{gcm}^{-3}\right]$ & $\Sigma\left[\mathrm{gcm}^{-2}\right]$ \\
\hline$C_{i}$ & 169.8 & $5.7 \times 10^{14}$ & $3.4 \times 10^{-13}$ & 393.1 \\
\hline & \multicolumn{4}{|c|}{$\bar{R}$ Regime $3 R_{3}=50 A U$} \\
\hline $\mathrm{Q}$ & $T[\mathrm{~K}]$ & Height $[\mathrm{cm}]$ & $\rho\left[\mathrm{gcm}^{-3}\right]$ & $\Sigma\left[\mathrm{gcm}^{-2}\right]$ \\
\hline$C_{i}$ & 814.5 & $1.12 \times 10^{14}$ & $4.1 \times 10^{-12}$ & 907.6 \\
\hline & \multicolumn{4}{|c|}{ Regime $4 R_{4}=10 \mathrm{AU}$} \\
\hline $\mathrm{Q}$ & $T[\mathrm{~K}]$ & Height $[\mathrm{cm}]$ & $\rho\left[\mathrm{gcm}^{-3}\right]$ & $\Sigma\left[\mathrm{gcm}^{-2}\right]$ \\
\hline$C_{i}$ & 1613.2 & $1.4 \times 10^{13}$ & $1.8 \times 10^{-10}$ & 5126.4 \\
\hline & \multicolumn{4}{|c|}{$\overline{\text { Regime } 5 R_{5}=6 \mathrm{AU}}$} \\
\hline $\mathrm{Q}$ & $T[\mathrm{~K}]$ & Height $[\mathrm{cm}]$ & $\rho\left[\mathrm{gcm}^{-3}\right]$ & $\Sigma\left[\mathrm{gcm}^{-2}\right]$ \\
\hline$C_{i}$ & 1918.3 & $7.1 \times 10^{12}$ & $6.5 \times 10^{-10}$ & 9282.8 \\
\hline & \multicolumn{4}{|c|}{ Regime $6 R_{6}=3 \mathrm{AU}$} \\
\hline $\mathrm{Q}$ & $T[\mathrm{~K}]$ & Height $[\mathrm{cm}]$ & $\rho\left[\mathrm{gcm}^{-3}\right]$ & $\Sigma\left[\mathrm{gcm}^{-2}\right]$ \\
\hline$C_{i}$ & 2767.9 & $3.0 \times 10^{12}$ & $3.0 \times 10^{-9}$ & $1.8 \times 10^{4}$ \\
\hline
\end{tabular}

Table 31).

\subsubsection{Disk Self-Sublimation of dust}

For our massive stellar disk model, we define that radius where the disk temperature reaches $1500 \mathrm{~K}$ as disk self-sublimation radius. Essentially, the disk temperature is determined by $\dot{M}$, while the radial velocity is governed by $\alpha$. The higher the accretion rate, more the gravitational potential energy from the accreted mass is converted to thermal energy in the inner region, thus increasing the disk temperature. High mass accretion rates $\sim 10^{-3}-10^{-4} \mathrm{M}_{\odot} \mathrm{yr}^{-1}$ are indirectly related to the formation of massive stars, indicating hotter disks in massive stars.

The dust sublimation radius can be estimated from the temperature profile in regime 4 (see Table (2) as the critical value of $1500 \mathrm{~K}$ is obtained in this regime,

$$
\begin{array}{r}
T=1.6 \times 10^{3} \mathrm{~K} \alpha^{-0.058}\left(\frac{\dot{M}}{10^{-3} \mathrm{M}_{\odot} \mathrm{yr}^{-1}}\right)^{0.101} \\
\left(\frac{M}{\mathrm{M}_{\odot}}\right)^{0.080}\left(\frac{r}{10 \mathrm{AU}}\right)^{-0.239}(.13)
\end{array}
$$

Thus the dust self-sublimation radius is

$$
\begin{array}{r}
R_{\text {sub }, \mathrm{disk}}=11 \mathrm{AU} \alpha^{-0.242}\left(\frac{\dot{M}}{10^{-4} \mathrm{M}_{\odot} \mathrm{yr}^{-1}}\right)^{0.422} \\
\left(\frac{M}{10 \mathrm{M}_{\odot}}\right)^{0.3347}\left(\frac{T}{1500 \mathrm{~K}}\right)^{-4.184}(14)
\end{array}
$$

\subsubsection{Dust Sublimation by Stellar radiation}

The second process of disk heating is by radiation from the central star. The dust sublimation radius due to the absorption of UV radiation from the star by the disk is dependent on the effective temperature and the radius of the star or equivalently to the luminosity of the star. We can estimate the relation between the temperature of the disk and the temperature of the star just by equating the flux from the star that is absorbed by the disk to the flux emitted by it considering it as black body. The disk is also assumed to be locally isothermal, which is an appropriate assumption for optically thick disks The mid plane temperature of the disk due to heating from central star can be 
estimated,

$$
T_{d}=\left(\frac{\theta \psi_{s}}{2 \psi_{i}}\right)^{1 / 4}\left(\frac{R_{*}}{r}\right)^{1 / 2} T_{*}
$$

(e.g. Dullemond et al. 2001) where $\theta$ is the angle with which the radiant flux is incident on the flaring disk, $\psi_{s}$ denotes the fraction of flux that is absorbed by the interior and $\psi_{i}$ is correction factor which accounts for the fact that the disk interior is not fully optically thick for its emission. The small correction due to backwarming in the disk is neglected. This correction mainly depends on the underlying dust properties (Monnier \& Millan-Gabet 2002). For a dust sublimation temperature of $\sim 1500 \mathrm{~K}$, we can estimate the sublimation radius due to absorption of radiation from the above equation (15) noted as Measure A whereas Measure B we denote as the sublimation radius calculated using the standard formula given by Monnier \& Millan-Gabet (2002). In Table 3 we show the typical values of the sublimation radius from these measures. For a typical B2 star of mass $=10 \mathrm{M}_{\odot}$ and $T_{\text {eff }}=22000 \mathrm{~K}($ Lang 1992 ) with ZAMS value for the stellar radius as $6 \mathrm{R}_{\odot}$, one gets a dust sublimation radius due to heating from stellar radiation of $R_{\mathrm{sub}, *} \sim 4 \mathrm{AU}$ for measure $\mathrm{A}$ and $\sim 3 \mathrm{AU}$ for measure $\mathrm{B}$. This value may increase by a factor of 2 considering the bloating star model (Hosokawa \& Omukai 2008), where the star bloats up to $100 \mathrm{R}_{\odot}$ and the effective temperature reduces to $5000 \mathrm{~K}$.

In Table 3, we compare for different stellar mass with typical order of mass accretion rate, the sublimation radius due to self heating from the disk and that due to heating from the star. This values are estimated for two different values of $\alpha=0.1,1$. It is evident from Table 3 that the dust sublimation radius due to heating from disk is a weak function of $\alpha$. Table 3 further indicates a ratio $R_{\text {sub,disk }} / R_{\text {sub } *} \sim 3$ as a good estimate over a wide range of mass accretion rates and stellar mass.

The value of $\alpha$ is related to the radial transport of matter in the disk, thus the mass accretion rate is a function of $\alpha$. For Table 3, the values of the sublimation radius is estimated using eq (13) assuming the same mass accretion rate for different $\alpha$ values. These are representative values of sublimation radius for typical mass accretion rates found in massive star forming regions. Using these values we optimize the ratio of the self sublimation radius of the disk to that caused by heating from stellar luminosity is around three. With this assumption, we obtain a relation of $\alpha$ and $\dot{M}$ for a particular stellar mass. This is obtained by setting $T=1500 \mathrm{~K}$ in the equation (13) and the radial distance as $3 \times R_{\text {sub }, *}$.

This relation can be used to have constraints on the mass accretion rates for a particular stellar mass. The plot for mass accretion rates with $\alpha$ is shown in Fig. 2 for typical OB type stars. If one considers the value of $\alpha$ as fixed to 1 , then from the Fig. 2 for the $10 M_{\odot}$ star one gets the accretion rate of $4.2 \times 10^{-4} M_{\odot} \mathrm{yr}^{-1}$, which implies that for this order of mass accretion rate the dust in the disk will sublimate at a distance of $3 \times R_{\mathrm{sub}, *}$. This clarifies that for the typical high accretion rates required for formation of high mass stars, the heating in the disk is very efficient to sublimate most of the dust grains in the midplane before the radiation could have any major effect on them.

The implication of this result is profound, as it demonstrates that viscous heating of the disk is the dominant mechanism in the midplane for sublimation of the dust. The self-sublimation in the turbulent massive disk sublimates most of the dust grains well before the stellar radiation could affect them. Essentially, this implies that more matter (in form of gas) can reach closer to the central star.

However, stellar radiation can have significant effects on the surface layer of the disk. The radial distance beyond which the stellar irradiation will dominate can be estimated by equating the flux from central star to the flux emitted from the disk. The flux from the disk is dependent on the effective surface temperature, which can be substantially lower than the midplane temperature depending on the optical depth. (see $\S 4.3)$

\subsection{Inner Gaseous disk structure}

In order to model the inner gaseous region of the disk, we consider the OPAL opacity tables applicable for higher temperatures (Iglesias \& Rogers 1996). The various dynamical quantities in the disk obtained using the opacity from the table and their comparison with the various analytical models are shown in Fig. 3. The variation of the opacity, used for the present work, from the opac- 
Table 3: Comparison of the sublimation radius due to heating in disk [equations (13) \& (14)] and due to heating from the star [equation (15)]. The temperature of the star and its radius are taken from Lang (1992). (see $§ 4.2$ for details)

\begin{tabular}{ccccccc}
\hline \hline Typical $\dot{M}\left[\mathrm{M}_{\odot} \mathrm{yr}^{-1}\right]$ & Spectral Type & Stellar Mass $\left(\mathrm{M}_{\odot}\right)$ & \multicolumn{2}{c}{$R_{\text {sub }, *}[\mathrm{AU}]$} & \multicolumn{2}{c}{$R_{\text {sub }, \text { disk }}[\mathrm{AU}]$} \\
\hline & & & Measure A & Measure B & $\alpha=0.1$ & $\alpha=1$ \\
\hline $10^{-5}$ & B5 & 5.9 & 1.4 & 1.0 & 6.1 & 3.5 \\
$10^{-4}$ & $\mathrm{~B} 2$ & 10 & 4.2 & 3.0 & 19.4 & 11.1 \\
$10^{-3}$ & $\mathrm{O} 6$ & 37 & 24.5 & 17.3 & 79.4 & 45.3 \\
\hline \hline
\end{tabular}

ity tables with the midplane temperature in the disk is shown in Fig. 4

The midplane temperature reaches very high values of the order of $10^{5} \mathrm{~K}$ at $20 \mathrm{R}_{\odot}$ (See Fig. 3 ). The radial profile for the temperature also shows a sudden break around $1 \mathrm{AU}$. This is related to the sudden rise in opacity around $3000 \mathrm{~K}$ as demonstrated in Fig. 4. Since the surface density and the mass density are inversely proportional to the temperature (see equations (10), (3), (5) \& (7)), their radial profiles show a sudden fall at that distance. Similar kind of sudden rise and fall can be seen also in the analytical results of Bell \& Lin (1994) which are we show alongside in Fig 3 .

We also compare with analytical radial profiles obtained using opacities as given by Ruden \& Pollack (1991). However these profiles just extent to the temperature range where the dust just sublimates and the opacity drops to a small value. These models fit very well in the outer disk region with the model described in the present work.

The high temperature upto $10^{5} \mathrm{~K}$ would imply a high ionization fraction in the disk close to the massive star. Thus the ionized gas could well couple to the large scale magnetic fields from ambient medium which is dragged by accretion and may give rise to collimated outflows.

In Fig [3, the variation of the scale height is also shown with the radial distance. Similar to radial profiles of other dynamical quantities, we get jump also in the profile of the scale height. This jump is quite interesting when dealing with disks around massive stars. The sudden rise of disk height around $1 \mathrm{AU}$ would help to shield outer disk regions from the radiation of central source. This affects the direction of radiation and leading to some sort of anisotropy in the radiation field.
We also investigate the radial profiles of the dynamical quantities for higher mass stars. These profiles are shown in Fig. 6. The midplane temperature profiles clearly show that as the central mass and accretion rate increase, the midplane temperature also increases and so the self sublimation radius will move further out. For instance, with central mass as $23 \mathrm{M}_{\odot}$ and accretion rate of $4 \times 10^{-3} \mathrm{M}_{\odot} \mathrm{yr}^{-1}$, the dust sublimates at $\sim 30 \mathrm{AU}$ whereas the dust sublimation radius is around $41 \mathrm{AU}$ for a $37 \mathrm{M}_{\odot}$ star with disk accretion rate of $8.5 \times 10^{-3} \mathrm{M}_{\odot} \mathrm{yr}^{-1}$. These parameters are also chosen with the same argument as used for $10 \mathrm{M}_{\odot}$ star. Also, the temperature in the inner most region is much higher for massive young star with higher mass. The mass density and the surface density profiles also show the same trend of increment in the inner region with increase in mass of central object.

On might question the thin disk approach to study the disk around massive young stars. However, with this approach we find that the disk around high mass stars are very similar to that around cataclysmic variables. The spectral signatures of disk around these compact objects were very well explained by this thin disk model. The opacity values from the OPAL opacity table are used to study the boundary layer of the white dwarfs (Collins et al. 1998). They obtain a very high central temperature near to the white dwarf but on applying the optical depth consistently from the table, they get the same order of magnitude of the effective temperature

$$
T_{\mathrm{eff}}^{4}=\frac{8 T^{4}}{3 \tau}
$$

as it is on the surface of a typical white dwarf.

In our case, the midplane temperature profile 
shows that the region very near $\left(\sim 20 \mathrm{R}_{\odot}\right)$ is very hot gas of $10^{5} \mathrm{~K}$, but to compare with the stellar surface effective temperature $\left(T_{\text {eff }}=22000 \mathrm{~K}\right.$ for $\left.M=10 \mathrm{M}_{\odot}\right)$ one has to take into account the finite optical depth and then the surface temperature of the disk, plotted in the same figure matches the surface temperature of the star at the very innermost regions. Thus the effective temperature of the disk can be considerably lower than the midplane temperature. The flux from the disk and the star are equated in order to compute the radial distance where the stellar irradiation can affect the disk surface,

$$
\frac{L_{\star}}{4 \pi r^{2}}=\sigma T_{\mathrm{eff}}^{4}
$$

where $L_{\star}$ is the luminosity of the star and $\sigma$ is the Stefan-Boltzmann constant. We find for a $10 \mathrm{M}_{\odot}$, the stellar irradiation will have a effect on the surface layers around $\sim 30 \mathrm{AU}$ for $\alpha=1$. If the $\alpha$ value is lowered, the stellar irradiation can have affect on the surface at even smaller radial distance of around 10 AU. However this effect will not alter the dust sublimation radius in the disk.

\subsection{Growth of Massive Stellar Embryo}

One of the major problems in the formation of massive stars is the large UV radiation from the protostars that exert pressure on the matter inhibiting the infall on them. This is because as the central mass increases also the central luminosity increases, thus exerting a large radiation pressure which is usually thought to halt the matter falling on the star. In order to get away with the large radiant flux and to form more massive stars, Yorke (2004) lists some of the favorable conditions such as $i$ ) Reduction of $\left.\kappa_{\text {eff }} i i\right)$ Reduction of effective Luminosity and iii) Increasing the gravitational acceleration.

\subsubsection{Reduction of $\kappa_{\mathrm{eff}}$ and effective Luminosity}

In earlier approaches considering the conditions for the formation of massive stars, the matter was thought to be accumulated by spherical accretion (Kahn 1974; Wolfire \& Cassinelli 1987). Using detailed dust models and complex grain size distribution, these models were able to put a limit on the maximum stellar mass of $20 \mathrm{M}_{\odot}$. In general, the dominance of the radiation pressure over gravity of the spherical infall of matter puts a strong constraint on the value of the opacity. In this case, the necessary condition to form a massive star is

$$
\frac{\kappa_{\mathrm{eff}} L}{4 \pi r^{2}}<\frac{G M_{*}}{r^{2}},
$$

(e.g. Yorke 2004; Zinnecker \& Yorke 2007), which implies an upper limit to the effective opacity,

$$
\kappa_{\text {eff }}<130 \mathrm{~cm}^{2} \mathrm{~g}^{-1}\left(\frac{M}{10 \mathrm{M}_{\odot}}\right)\left(\frac{L}{1000 \mathrm{~L}_{\odot}}\right)^{-1}
$$

In the disk scenario, there would be additional contribution to the forces mentioned in equation (17), such as disk gas and ram pressure.

In the present work, we demonstrate that most of dust, due to the self sublimation of accretion disk, is destroyed already at distances larger that $\sim 10 \mathrm{AU}$ from the central source which helps to reduce the $\kappa_{\text {eff }}$. It is evident that the maximum value of the opacity even in the innermost region of the disk is less than $5 \mathrm{~cm}^{2} \mathrm{~g}^{-1}$ (see Fig. 4), which is much smaller than the upper limit given by equation (18) required for the formation of a typical massive star. This suggests that the radiation pressure is no longer inhibiting the accretion process onto to the central star and thus allowing more massive stars to form. This effect is aiding the matter to flow closer to the central object but does not ensure that matter will be accreted on the central star. Thus reduction in opacity is an essential requirement but it does not guarantee growth of mass as the isotropic radiation from the star may still stop the dust by exerting the pressure which is dependent on the luminosity of the central object.

A sketch of our model calculations assuming a $10 \mathrm{M}_{\odot}$ central star is shown in Fig. 7 . The scaling of the figure is done using a fiducial jet radius. The main components in the figure are the dusty disk which is feeded with matter by the core, the inner gaseous disk and the large scale bipolar outflows. The region near to the dust sublimation radius shows the presence of dust and gas and large variation of opacity is seen in this region. Very close to the source there is large flux of UV and visible radiation which is blocked from the midplane of outer dusty disk by the high optical depths $(\tau \gg 1)$ of the inner gaseous species. The figure also shows the possibility of radiation flux escaping more in the direction perpendicular to the plane. This "flashlight effect" was introduced 
by Yorke \& Bodenheimer (1999) in order to have anisotropy in the radiation and allow matter to accrete.

\subsubsection{Overcoming Radiation Pressure}

The reduction of effective opacity and luminosity ensures the matter to come closer to the central star. In the region very near to the star matter is subjected to different pressure sources, all of them play an important role in understanding the dynamics of disk accretion under the influence of stellar radiation pressure. We therefore compare the contribution of different pressure sources, considering typically a $10 \mathrm{M}_{\odot}$ star.

The radiation pressure from the star is a function of the radial distance from the surface of the star,

$$
\begin{gathered}
P_{\text {rad }, \star}(r)=\frac{L_{\star}}{4 \pi c r^{2}} \\
=5.9 \times 10^{2} \operatorname{ergcm}^{-2}\left(\frac{T_{\text {eff }}}{22000 \mathrm{~K}}\right)^{4}\left(\frac{r}{\mathrm{R}_{\star}}\right)^{-2}
\end{gathered}
$$

The ram pressure from the disk counteracts the radiation force from star and will allow the matter to accrete. We estimate the ram pressure $P_{\text {ram }}=\rho v_{\mathrm{r}}^{2}$ using our consistent dust and gas opacity model (see $\S 4.1$ and $\S 4.3$ ),

$$
\begin{array}{r}
P_{\text {ram }}(r)=2.08 \times 10^{3} \operatorname{ergcm}^{-2}\left(\frac{\rho}{2.32 \times 10^{-9} \mathrm{gcm}^{-3}}\right)^{-1} \\
\left(\frac{\dot{M}}{10^{-4} \mathrm{M}_{\odot} \mathrm{yr}^{-1}}\right)^{2}\left(\frac{M}{10 \mathrm{M}_{\odot}}\right)\left(\frac{T}{5.03 \times 10^{4} \mathrm{~K}}\right)^{-1} \\
\left(\frac{\mathrm{R}_{\star}}{6 \mathrm{R}_{\odot}}\right)^{-5}\left(\frac{r}{10 \mathrm{R}_{\star}}\right)^{-5}
\end{array}
$$

Figure 8 shows the radial profiles of both pressure sources. The figure clearly depicts that the radiation pressure from the star is much below the ram pressure in the disk which will aid the matter to overcome radiation pressure and accrete on the central star. In the very inner region of the disk, the disk radiation pressure becomes comparable to the gas pressure in the disk which may result in thermal instability.(Figure 8)

\section{Stability of Disks}

The disks around massive stars can be unstable due to axisymmetric gravitational instability.
They can also be unstable due to fragmentation due to rapid cooling of the disk as compared to the dynamical time scale. Gammie 2001; Rice et al. 2003; Rafikov 2005). We will also see that these disks can be also unstable thermally in the very inner region, close to the massive star.

\subsection{Gravitational Instability}

The criterion for the gravitational instability of a disk is given by the Toomre parameter

$$
Q=\frac{c_{s} \Omega}{2 \pi G \Sigma}
$$

with $\Omega$ as the Keplerian angular velocity and $\Sigma$ as the surface density of the disk (Toomre 1964). For $Q>1$ the disk is stable (no fragmentation), while $Q<1$ leads to instability in the disk. Physically, the Toomre parameter can be considered as the ratio of centrifugal force along the radial direction to the gravitational force acting in the direction perpendicular to the radial motion. Thus, if a local accumulation of mass is moving in a certain orbit, then this would lead to slowing down of orbital motion and also more gravitational force acting downward which implies that the value $\mathrm{Q}$ will decrease and eventually when $Q<1$ the downward gravity force wins and leads to instability generating overdense regions. 7

It has been known from simulations (Krumholz et al. 2007, 2009) and observations in case of G 192.163.82 (Shepherd et al. 2001, considering large observational errors) that disks in massive stars are unstable as they may have $\mathrm{Q}$ value low and sometimes $<1$ for some radial extent.

The Toomre parameter decreases with the radial distance, and at some radial distance and the disk becomes gravitational instable. Figure 4 shows the radial behavior of the Toomre parameter in case of typical disk parameters $(\dot{M}=$ $4.2 \times 10^{-4} \mathrm{M}_{\odot} \mathrm{yr}^{-1}$, stellar mass $M=10 \mathrm{M}_{\odot}$ and $\alpha \sim 1$ ). It is clear that such a disk is unstable as $Q<1$ after $\sim 100 \mathrm{AU}$.

\footnotetext{
${ }^{\ddagger}$ The Toomre criteria can be written as a product of two ratios - $\frac{E_{\text {thermal }}}{E_{\text {grav }}} \frac{E_{\text {rot }}}{E_{\text {grav }}}<1$. If only one of the ratios is less than unity, that will not guarantee that fragmentation will occur.(see $\S 5.2$ )
} 


\subsection{Fragmentation of Disks}

Disks which are stable for axisymmetric gravitational instability may not be necessarily stable for fragmentation which leads to formation of bound objects. Gravitational instability sets an upper limit of the sound speed where as the analysis for fragmentation and cooling time sets a lower limit on the speed of sound in the disk (Rafikov 2005).

Numerical simulations Gammie 2001; Rice et al. 2003 ) and analytical solutions (Rafikov 2005) suggest that for a disk to avoid fragmentation, the cooling time scale should be larger than the dynamical timescale $\left(t_{d y n} \sim \Omega^{-1}\right)$. The threshold relation of the cooling time can be obtained, $\Omega t_{\text {cool }}<\zeta$, where the factor $\zeta \approx 3$, as obtained from simulations using constant $t_{\text {cool }}$ Gammie 2001; Rice et al. 2003). For the present purpose, we apply cooling time as given by Rafikov (2005),

$$
t_{\text {cool }} \approx \frac{\Sigma c_{s}^{2}}{\gamma-1} \frac{f(\tau)}{2 \sigma T^{4}}
$$

where $f(\tau)=\tau+\frac{1}{\tau}$ describes the efficiency of cooling and depends on the optical depth $\tau$. In the above equation (21), $\gamma$ is the adiabatic index and factor $\gamma-1$ considered to be of the order of unity (Rafikov 2005).

The variation of the cooling time, as given by eq. (21), with radial distance is shown in Fig. 4 for different values of $\alpha$. These curves are made applying the Bell \& Lin opacity power laws. The solid line in the figure for cooling time determines the threshold for fragmentation to set in. The relation suggests that a value $\alpha \sim 1$ would lead to a disk which may fragment completely. However, the lower values of $\alpha \sim 0.1,0.01$ would enable a stable disk against fragmentation. The value $\alpha \sim 0.1$ is consistent with that obtained by observational modelling of ionized disks (King et al. 2007).

\subsection{Thermal Instability}

Thin accretion disks around black holes which are supported by radiation pressure (region A, See §2) were found to be thermally instable (Shakura \& Sunyaev 1976). The physical reason for such kind of instability is inefficient cooling in the disk as compared to the viscous heating. This leads to overheating which causes expansion which in turn overheats the disk eventually leading to a thermal runaway. There were ideas of application of slim disk model to this region, which is a stable branch in the S-shaped $\dot{M}-\Sigma$ curve, because such a model prevents the radiation to escape from the disk and allowing the flux to be advected along with the flow of matter (Abramowicz et al. 1988).

In the modelling of the disk around massive star, we see that the radiation pressure becomes comparable to the gas pressure in the very innermost region of the disk $\leq 4 R_{\star}$ for a $10 \mathrm{M}_{\odot}$ star. Here the assumption that $P_{\text {gas }}>P_{\text {rad }}$ no longer holds, and we stop our iterations at this radii. However, the application of concept of slim disk in protostellar disks for efficient cooling is not really probable. This is because of the fact that advected flux should get rid in some manner, it is viable in disks around black holes as the flux can be advected into the black holes, however in case of disks around stars this flux will heat up the star, which may be not physical. Turner et al. (2007) applied the concept of photon bubble instabilities to the young massive stellar environments, which could be an efficient way of cooling the innermost region of massive star forming region as well.

\subsection{How big is $\alpha$ ?}

The value of $\alpha$ parameterizes the viscosity in the disk. In the present case, we see that higher value of $\alpha \sim 1$ ensures that the disk within $\sim$ $100 \mathrm{AU}$ is stable to axisymmetric gravitational instability, though for this high value of $\alpha$, the disk completely would fragment.

For $\alpha \sim 0.1$ the disk would become stable to fragmentation. In this case, the part of the disk stable to axisymmetric gravitational instability reduces to $\leq 80-90 \mathrm{AU}$

For $\alpha=0.01$, the disk would again be stable to fragmentation, but now most part of the disk is subjected gravitational instability. The effect of lowering the value $\alpha$ on the Toomre parameter can be seen in Fig. 4

The value of $\alpha$ is usually chosen as a parameter in disk modelling for different purposes. In case of low mass stars, the range of $\alpha$ values accepted is around 0.001-0.01, which is believed to be produced by magneto-rotational instability (Balbus \& Hawley 1998). In case of massive stars, the value of $\alpha$ can be higher by one order of mag- 
nitude and the physics involved to generate this high $\alpha$ value could be different from that seen in low stellar mass case. (for e.g. Gravitational instability)

A high $\alpha$ would imply that matter flows radially with high speed near to local sound speed and there could be a possibility of accretion shocks produced to make the radial speed of matter subsonic. These accretion shocks have been proposed for the close circumstellar environments of high mass protostar such as CRL 2136 with the $\mathrm{H}_{2} \mathrm{O}$ maser (Menten \& van der Tak 2004).

The other way of treating viscosity in the disk would be by assuming a radial profile, $\alpha(r)$, such that its value may be high $(\sim 1)$ in the outer region and lower $(\sim 0.1)$ in the inner region of the disk. Such a radial profile would then lead to fragmentation resulting in formation of bound objects influencing the structure in the outer part of the disk around most massive star formed such that each bound object may form itself a low mass or even high mass star (Krumholz et al. 2009). However, the inner ionized disk can be still stable and can launch large scale bipolar outflows.

In Fig. 5, variation of the dynamical quantities obtained from the present model for a $10 \mathrm{M}_{\odot}$ star with two different values of $\alpha$ is shown. Apart from changing the $\alpha$ value we also change the value of mass accretion rate according to the figure 2 , There is no considerable change in midplane temperature profile, which is due to the fact that by changing two parameters also bring change in the opacity and the cumulative effect cancels the variation in temperature 5 . However, this does not happen for mass density as the dependence on the above variations is different. Since the height depends only on the temperature, the height profile also does not show any variation.

\section{Implications for Observations}

Early stages of massive star formation are difficult to observe, as the whole process is enclosed in an envelope of dust. Also these high mass star forming regions are at a distance of few kilo parsecs which makes the observation more difficult due to limited resolution of the present day tele-

\footnotetext{
$\S$ Note that $\Sigma$ is inversely proportional to $\alpha$ and decreasing
} the $\alpha$ will increase density and thus opacity scopes.

One of the essential findings of the present work is the high temperature of the order of $\sim 10^{5} \mathrm{~K}$ in the inner disk. At such a high temperature, the opacity is mostly dominated by electron scattering and there is a pool of highly energetic electrons present close to the massive star. These electrons might be responsible for soft X-ray emissions from massive young stars.

There have been observations of X-rays in some of the massive young sources in the W3 complex (Hofner et al. 2002) and also in GGD 27 (Pravdo et al. 2009). In the case of GGD 27, observed hard X-rays emissions have energies of the order of 2-10 KeV. The temperatures obtained from present work indicate emissions of soft Xrays, however the soft $\mathrm{X}$-rays emissions have a large extinction in massive star forming regions. There might be possibility that these less energetic electrons are accelerated by magnetic fields giving rise to hard X-ray emission and also the hard $\mathrm{X}$-ray emission may come from shocks produced when the accreted matter falls on the surface of star (Montmerle 2007).

Amidst all the difficulties, observers were successful to resolve a fattened structure having length scales of $\sim 1000 \mathrm{AU}$. The best candidate of showing presence of a rotating Keplerian disk is IRAS $21026+4104$ (Cesaroni et al. 2005) which is predicted to be early B star (Mass $=7-12 \mathrm{M}_{\odot}$ ). They estimate the radial profile of mean kinetic temperature for the Keplerian disk and mass density profile using different lines. They obtain the best fit for temperature profile as $T(R) \propto R^{-q}$ where $\mathrm{q}$ is $0.57-0.75$ and that of mass density $\rho(R)=R^{-2.1}$ for $\mathrm{HCO}^{+}$(1-0) line. The values of the powers obtained are very close to the best fit of these profiles from the present model (Fig. 1). Schrever et al. (2006) also were able to resolve a rotating structure in an embedded 8-10 $\mathrm{M}_{\odot}$ massive star AFGL 490. They also obtain a Keplerian profile for the rotational velocity and the best fit radial power law for the surface density was $\Sigma \propto R^{-1.5}$, which is close to the value obtained from the present model $\left(\Sigma \propto R^{-1.1}\right)$. In case of low mass stars, usually the density in the disk is fitted using the gaussian as given by 
(Whitney et al. 2003; Wolf et al. 2008)

$$
\rho_{\text {disk }}=\rho_{0}\left(\frac{R_{*}}{r}\right)^{a} \exp \left\{\frac{-1}{2}\left[\frac{z}{h(r)}\right]^{2}\right\}
$$

where the scale height has the radial dependence of the form

$$
h(r)=h_{0}\left(\frac{r}{R_{*}}\right)^{b}
$$

The best fit obtained for the case of disks in "Butterfly" star was $\mathrm{a}=2.35, \mathrm{~b}=1.28$ (Wolf et al. 2008). These results are interesting as similar radial fits to the elongated continuum are obtained even for the recent observational results of young high mass source IRAS 18151-1208 (Fallscheer private communication). Also similar fits are obtained from the present model.

\section{Conclusion}

In this paper we have presented - for the first time - global models for accretion disks around massive young stars.

We have solved the thin disk equations taking into account the proper opacities for dust and gas. In particular we consider Rosseland mean dust opacities given by Ruden \& Pollack (1991) and the gas opacities from the OPAL opacity tables (Iglesias \& Rogers 1996).

This enables us to provide the dynamical quantities of disk accretion from the very inner part at radii of $0.1 \mathrm{AU}$ to the outer region of the disk at about $100 \mathrm{AU}$. At the same time this provides a theoretical link between outer disk which is accessible in principle by observations and the inner disk which is not yet possible to resolve observationally.

Our main results can be summarized as follows.

1. For typical stellar masses and accretion rates we find very high midplane temperatures of the order of $10^{5} \mathrm{~K}$ for radii less than $\sim 0.1 \mathrm{AU}$

2. Due to the high disk temperatures the dust sublimates already at distances which are about a factor three larger than caused by the stellar irradiation. This disk selfsublimation lowers the disk opacities considerably and allows for disk accretion in the stellar radiation field.
3. We estimate the stability of these disk by the Toomre criterion and find that our thin disks around e.g. a $10 \mathrm{M}_{\odot}$ star becomes gravitationally unstable beyond $100 \mathrm{AU}$. We also see for $\alpha$ values close to 1 , the disk would fragment completely whereas for $\alpha \sim 0.1$ the disk could remain stable to fragmentation. We also discuss the effect on the dynamics and stability of the disk model with the variation of $\alpha$.

4. For the given disk and stellar parameters and disk opacities we find that the stellar radiation pressure is negligible against the disk ram pressure and gas pressure and therefore cannot hinder accretion towards a massive young star.

Considering the high disk temperatures and the rather large disk viscosity parameter, disk around massive young stars seem to be intrinsically different from the low mass equivalents in particular to the different form of disk opacity

We also find that for higher mass stars with high accretion rates the dust sublimates further away and obtain much higher temperature for the same. The presence of the optically thick gaseous component and anisotropy of radiation prevents the matter to be unaffected by the radiation from the star. 

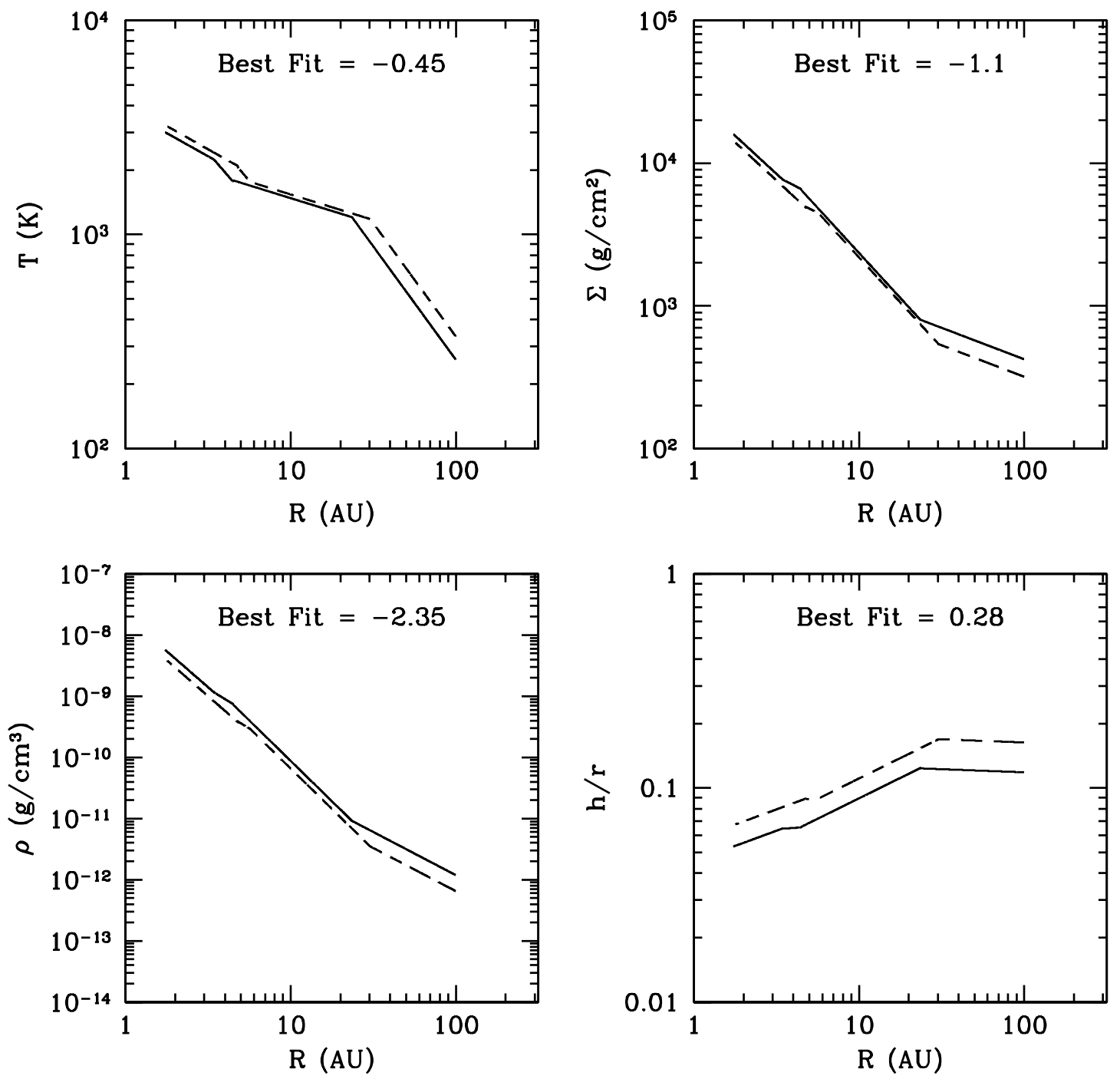

Fig. 1. - Radial profiles of midplane temperature $(\mathrm{T})$, mass density $(\rho)$, surface density $(\Sigma)$ and the scale height ratio $(h / r)$ for the outer dust dominated disk. The dashed line represents the tabulated values (see Table 2), the solid line is obtained by using the Ruden \& Pollack (1991) opacity power laws. These plots are for typical $\dot{M}=4.2 \times 10^{-4} \mathrm{M}_{\odot} \mathrm{yr}^{-1}$, stellar mass $M=10 \mathrm{M}_{\odot}$ and $\alpha \sim 1$. The radial index of the best fitted linear plot are mentioned in each subplot. 


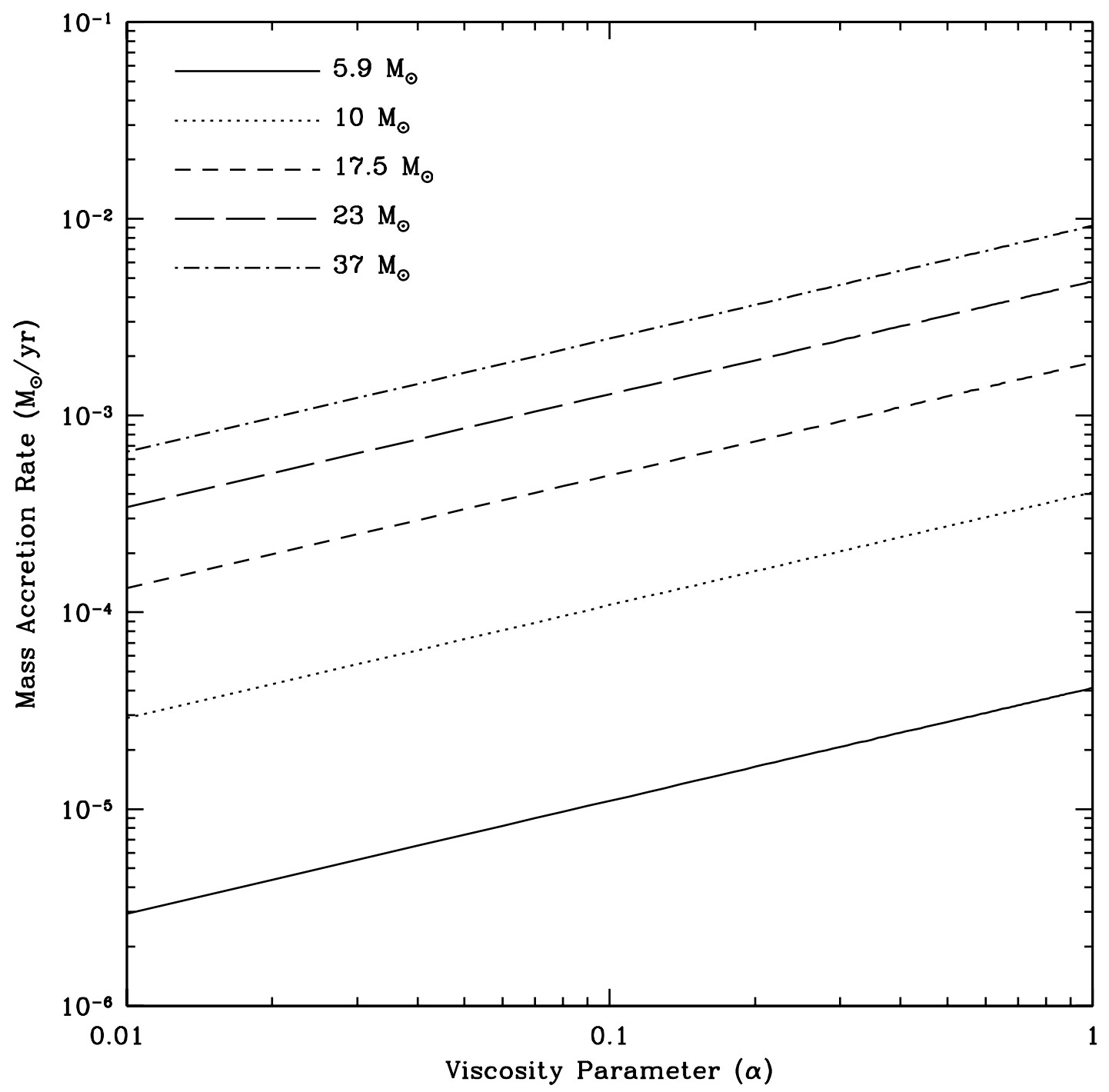

Fig. 2.- This plot shows the variation of mass accretion rate with the viscosity parameter so that the dust sublimation temperature due to viscous heating is around three times that of heating from star. The various lines are for different spectral type of stars (luminosity values obtained from Lang 1992). These plots indicate that mass accretion rate is a weak function of $\alpha$. 

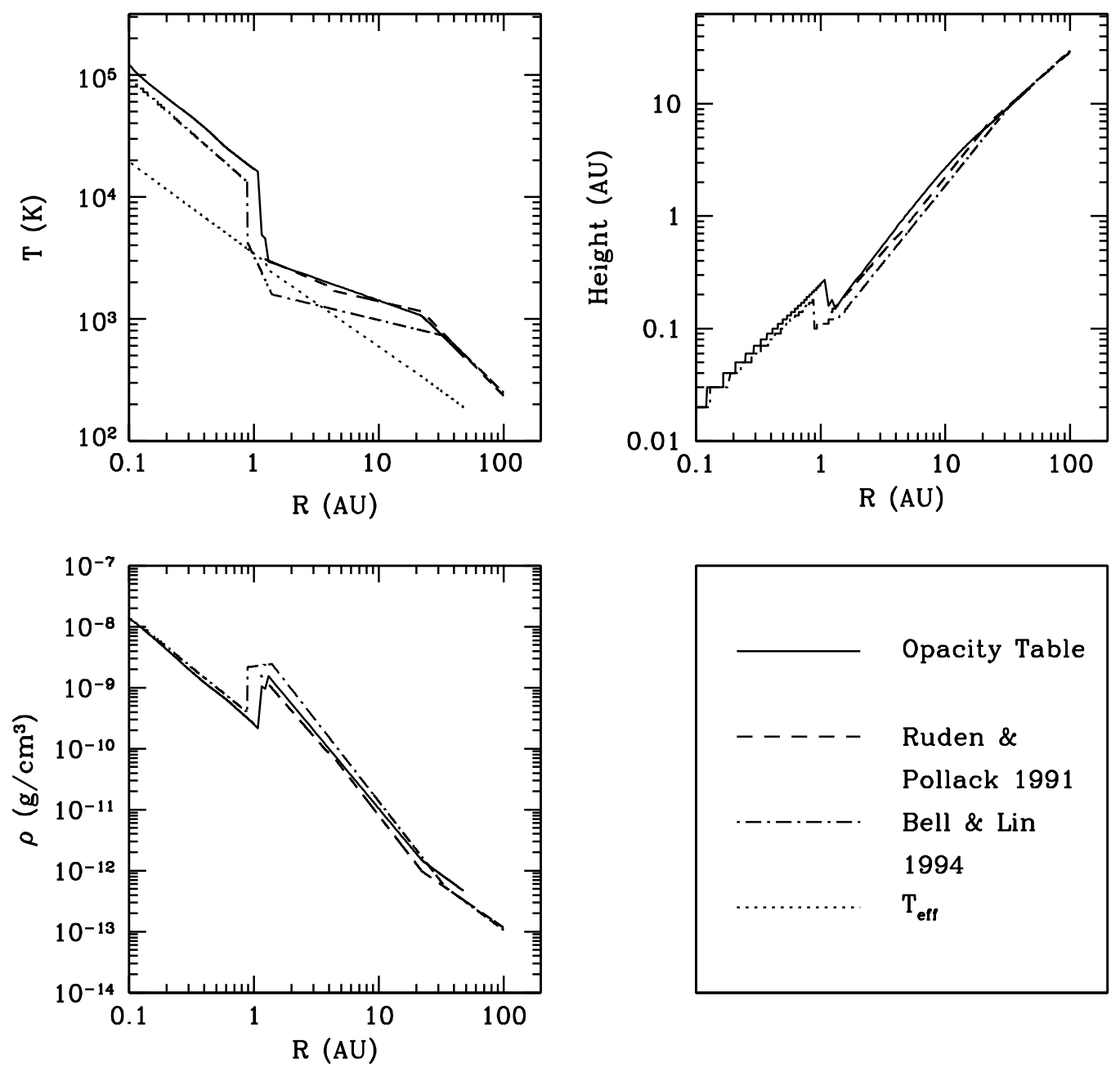

Fig. 3.- Radial profiles of temperature (T), mass density $(\rho)$, scale height of the disk extended to the inner region. The solid line represents the values from opacity table (Ferguson et al. 2005) and OPAL (Iglesias \& Rogers 1996), the dashed line is using the opacity power laws by Ruden \& Pollack (1991) and the dot-dashed line are the values for Bell and Lin opacity power laws. The dotted line shown in temperature profile represents the $T_{\text {eff }}$ profile with radius whereas the other lines are for the midplane temperature. These plots are for typical $\dot{M}=4.2 \times 10^{-4} \mathrm{M}_{\odot} \mathrm{yr}^{-1}$, stellar mass $M=10 \mathrm{M}_{\odot}$ and $\alpha \sim 1$ 

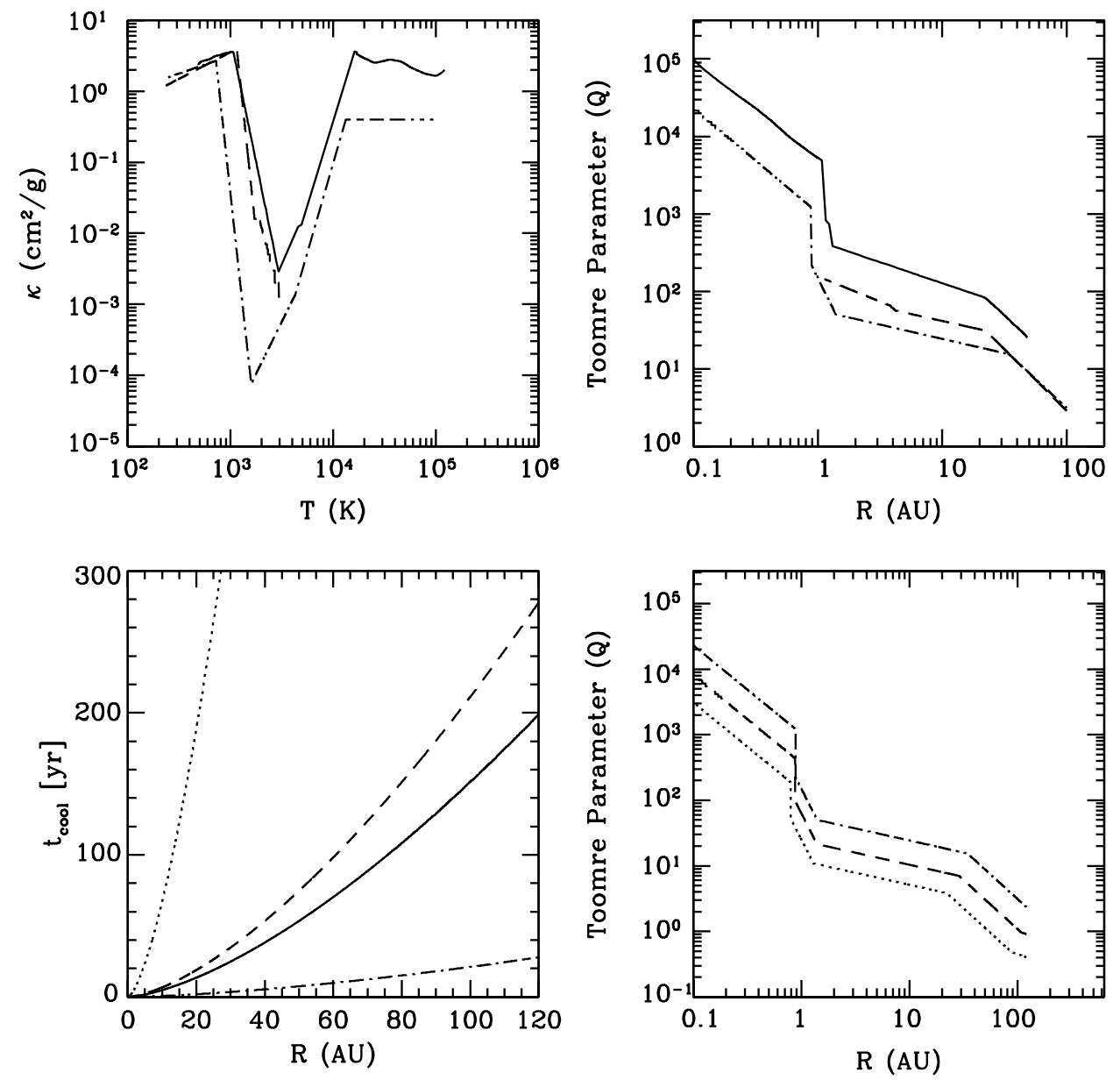

Fig. 4.- The opacity variation with temperature (top left panel), and the variation of Toomre parameter $\mathrm{Q}$ with the radial distance (top right panel). The solid line represents the values from opacity table (Ferguson et al. 2005) and OPAL (Iglesias \& Rogers 1996), the dashed line is using the opacity power laws by Ruden \& Pollack (1991) and the dot-dashed line are the values for Bell and Lin opacity power laws. The bottom panels shows how the variation of $\alpha$ values affect the cooling time and Toomre parameter, $\alpha=0.01$ is denoted by dotted line, where as $\alpha=0.1$ is dashed line and $\alpha=1.0$ is by the dot-dashed line. Solid line in the curve represents the threshold condition for the fragmentation to set in. 

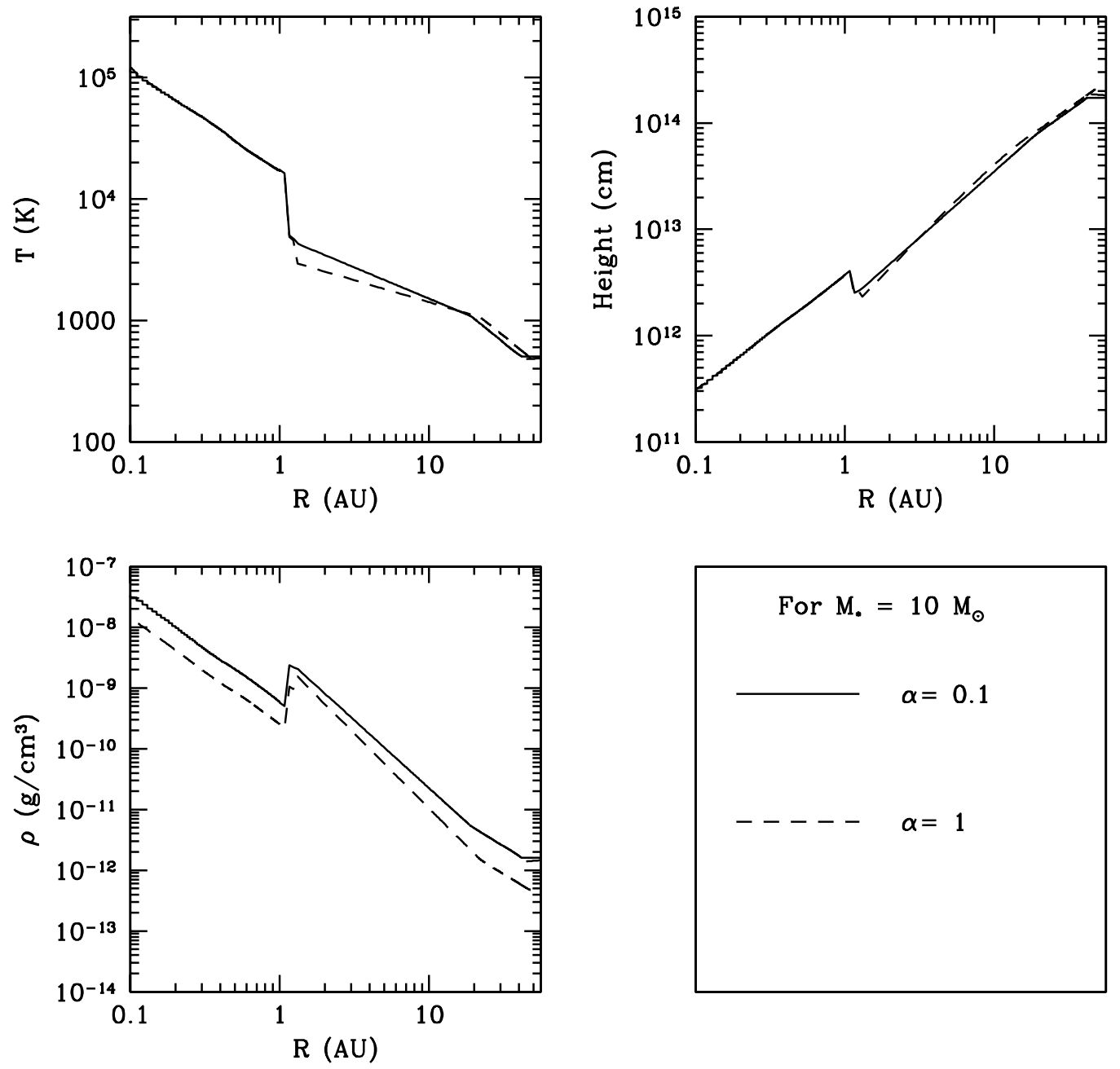

Fig. 5.- Dynamical quantities for two different $\alpha$ values. The solid line represents plot with $\alpha=0.1$ and $\dot{M}=10^{-4}$ where as dashed line is for $\alpha=1$ and $\dot{M}=4.2 \times 10^{-4}$ 

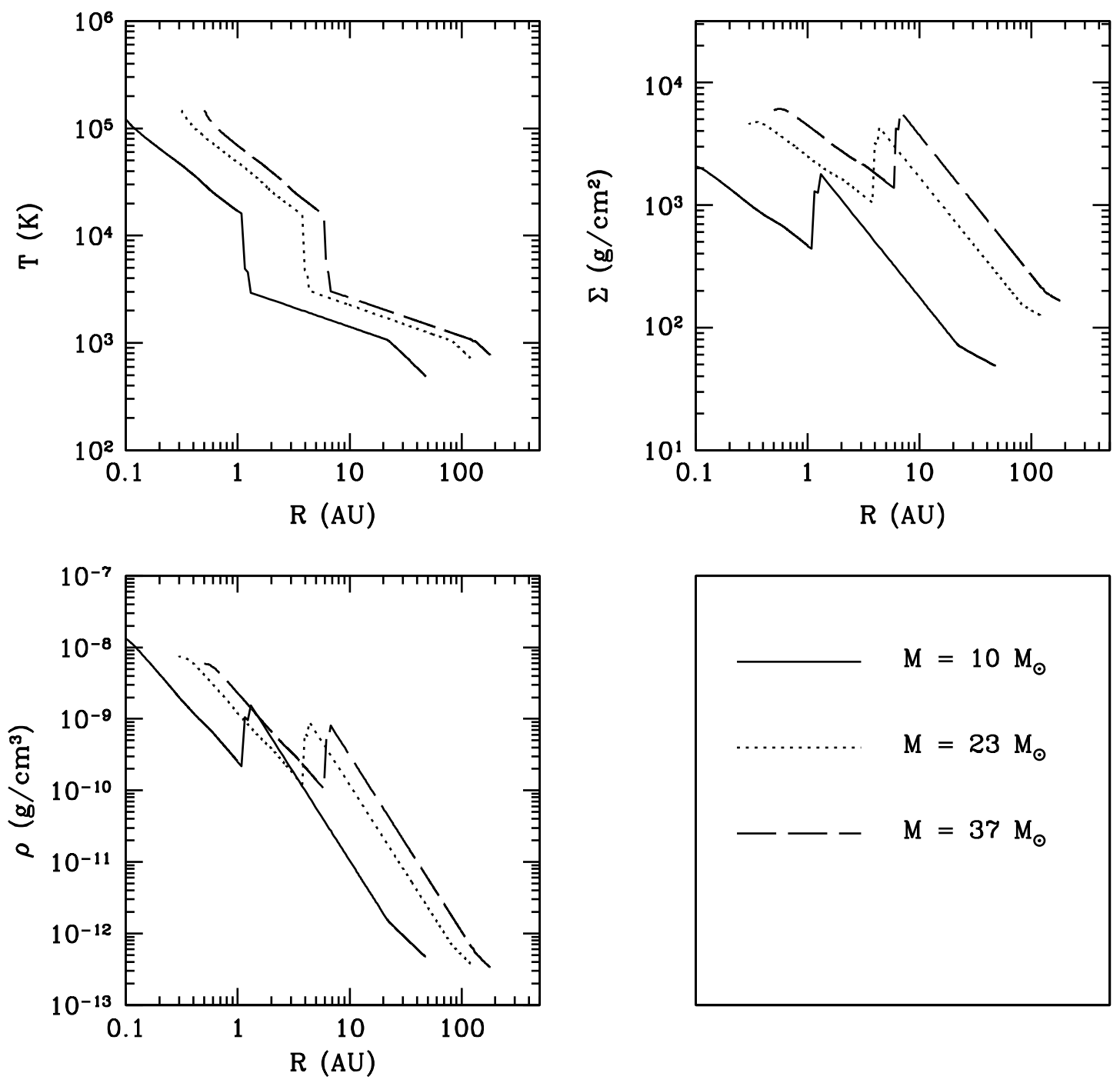

Fig. 6.- Radial profiles of temperature $(\mathrm{T})$, mass density $(\rho)$, surface density $(\Sigma)$ of the disk extended to the inner region. The solid line corresponds to $M=10 \mathrm{M}_{\odot}$, the dotted line for $M=23 \mathrm{M}_{\odot}$ and the dashed line is for central stellar mass $M=37 \mathrm{M}_{\odot}$. 


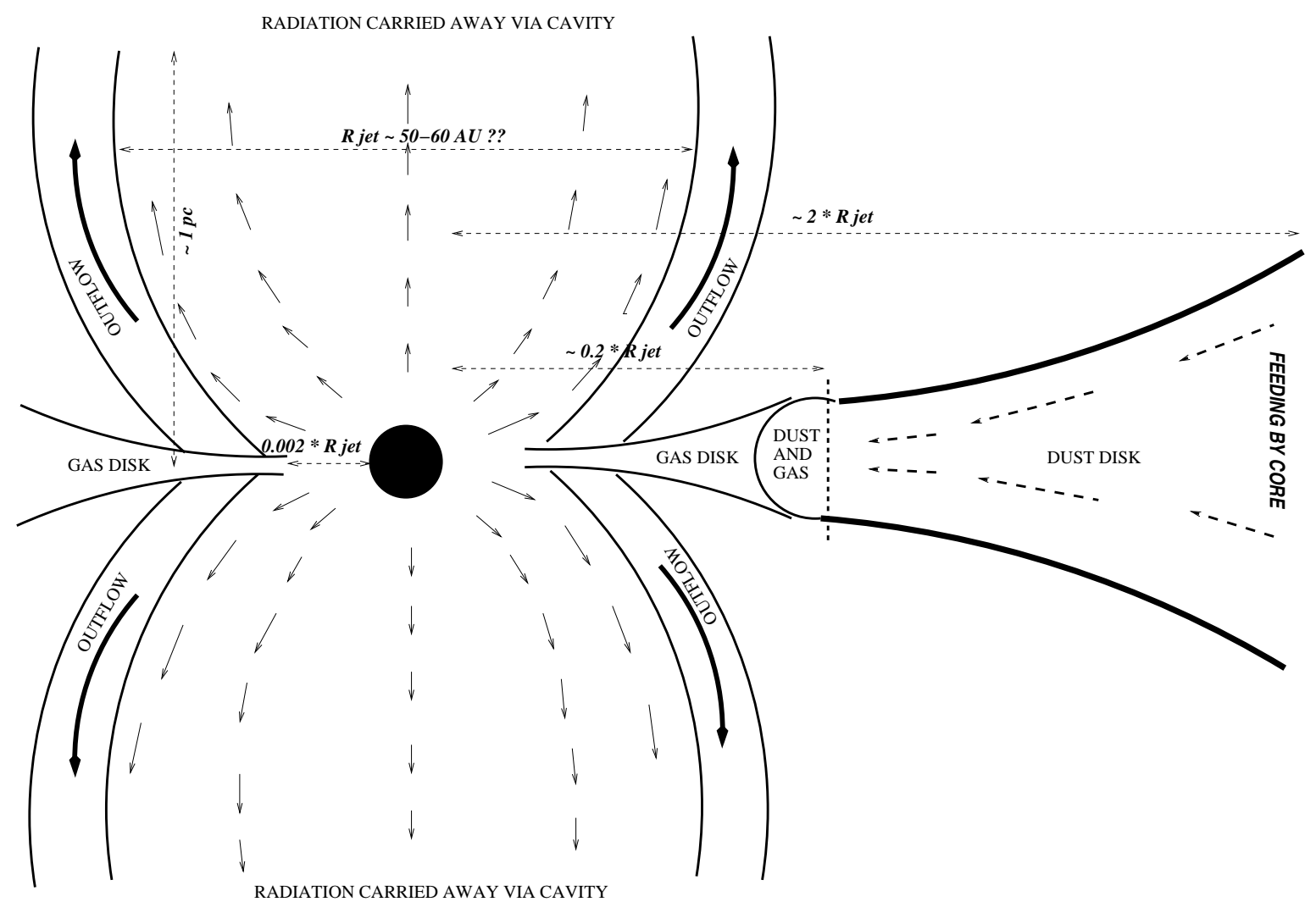

Fig. 7.- Pictorial representation of the inner region of the massive star. 


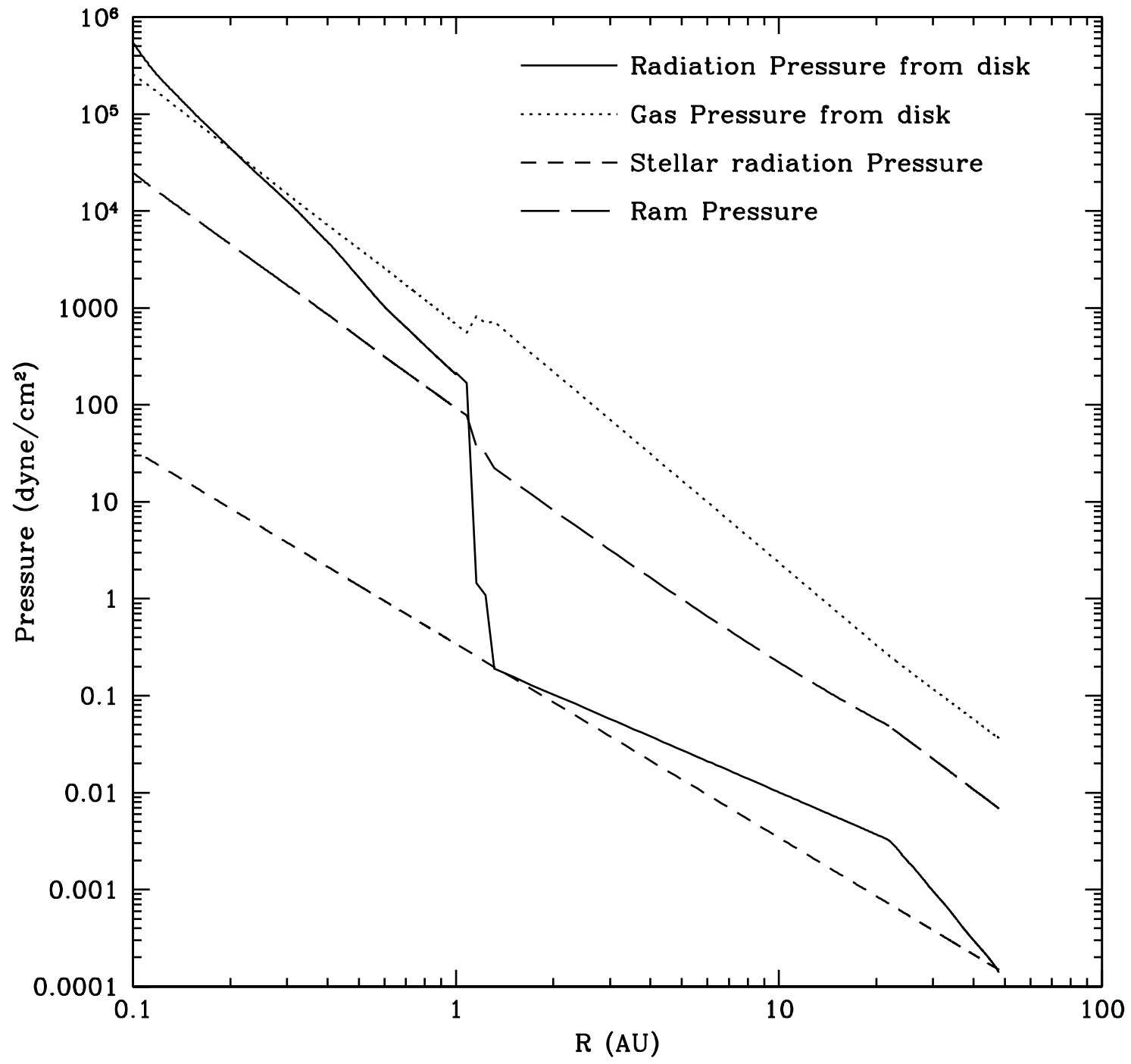

Fig. 8.- The above figure compares the pressure due to different sources in the circumstellar environment for a $10 \mathrm{M}_{\odot}$ star 
We acknowledge the Klaus Tschira Stiftung for funding this work carried out at Max Planck Institute of Astronomy, Heidelberg and also convey our thanks to the Heidelberg Graduate school of Fundamental Physics (HGSFP). We thank John Bally for his enlightening comments on this work and also the anonymous referee for his/her valuable comments concerning the stability analysis of the disk. We also like to thank S. Wolf, H. Linz, C. Dullemond and D. Semenov for their helpful comments and useful suggestions for this work. H.B. acknowledges financial support by the Emmy-Noether-Program of the Deutsche Forschungsgemeinschaft (DFG, grant BE2578). B.V. also thanks Surhud More and Mario Gennaro for their apt suggestions.

\section{REFERENCES}

Abramowicz, M. A., Czerny, B., Lasota, J. P., \& Szuszkiewicz, E. 1988, ApJ, 332, 646

Balbus, S. A. \& Hawley, J. F. 1998, Reviews of Modern Physics, 70, 1

Bell, K. R. \& Lin, D. N. C. 1994, ApJ, 427, 987

Beuther, H. 2007, arXiv:0712.1109B

Beuther, H., Schilke, P., Sridharan, T. K., Menten, K. M., Walmsley, C. M., \& Wyrowski, F. 2002, A\&A, 383, 892

Beuther, H. \& Shepherd, D. 2005, in Cores to Clusters: Star Formation with Next Generation Telescopes, ed. M. S. N. Kumar, M. Tafalla, \& P. Caselli, 105-119

Casse, F. \& Keppens, R. 2002, ApJ, 581, 988

Cesaroni, R., Neri, R., Olmi, L., Testi, L., Walmsley, C. M., \& Hofner, P. 2005, A\&A, 434, 1039

Collins, T. J. B., Helfer, H. L., \& van Horn, H. M. 1998, ApJ, 502, 730

D'Alessio, P., Canto, J., Calvet, N., \& Lizano, S. 1998, ApJ, 500, 411

Del Popolo, A. \& Ekşi, K. Y. 2002, MNRAS, 332, 485

Dullemond, C. P., Dominik, C., \& Natta, A. 2001, ApJ, 560, 957
Fendt, C. 2009, ApJ, 692, 346

Ferguson, J. W., Alexander, D. R., Allard, F., Barman, T., Bodnarik, J. G., Hauschildt, P. H., Heffner-Wong, A., \& Tamanai, A. 2005, ApJ, 623,585

Frank, J., King, A., \& Raine, D. 1992, Accretion power in astrophysics. (Camb. Astrophys. Ser., Vol. 21,)

Gammie, C. F. 2001, ApJ, 553, 174

Grave, J. M. C. \& Kumar, M. S. N. 2009, arXiv:0901.2053G

Helling, C., Winters, J. M., \& Sedlmayr, E. 2000, A\&A, 358, 651

Hofner, P., Delgado, H., Whitney, B., Churchwell, E., \& Linz, H. 2002, ApJ, 579, L95

Hosokawa, T. \& Omukai, K. 2008, in Astronomical Society of the Pacific Conference Series, Vol. 387, Massive Star Formation: Observations Confront Theory, ed. H. Beuther, H. Linz, \& T. Henning, 255

Iglesias, C. A. \& Rogers, F. J. 1996, ApJ, 464, 943

Kahn, F. D. 1974, A\&A, 37, 149

Keto, E. 2007, ApJ, 666, 976

King, A. R., Pringle, J. E., \& Livio, M. 2007, MNRAS, 376, 1740

Krumholz, M. R., Klein, R. I., \& McKee, C. F. 2007, ApJ, 656, 959

Krumholz, M. R., Klein, R. I., McKee, C. F., Offner, S. S. R., \& Cunningham, A. J. 2009, Science, 323, 754

Lang, K. R. 1992, Astrophysical Data I. Planets and Stars. (Astrophysical Data I. Planets and Stars, X, 937 pp. 33 figs.. Springer-Verlag Berlin Heidelberg New York)

Lin, D. N. C. \& Papaloizou, J. 1985, in Protostars and Planets II, ed. D. C. Black \& M. S. Matthews, 981-1072

Menten, K. M. \& van der Tak, F. F. S. 2004, A\&A, 414,289 
Monnier, J. D. \& Millan-Gabet, R. 2002, ApJ, 579, 694

Montmerle, T. 2007, in IAU Symposium, Vol. 243, IAU Symposium, ed. J. Bouvier \& I. Appenzeller, 23-30

Morfill, G. E. \& Wood, J. A. 1989, Icarus, 82, 225

Ossenkopf, V. \& Henning, T. 1994, A\&A, 291, 943

Pravdo, S. H., Tsuboi, Y., Suzuki, Y., Thompson, T. J., \& Rebull, L. 2009, ApJ, 690, 850

Pudritz, R. E., Ouyed, R., Fendt, C., \& Brandenburg, A. 2007, in Protostars and Planets V, ed. B. Reipurth, D. Jewitt, \& K. Keil, 277-294

Rafikov, R. R. 2005, ApJ, 621, L69

Rice, W. K. M., Armitage, P. J., Bate, M. R., \& Bonnell, I. A. 2003, MNRAS, 339, 1025

Ruden, S. P. \& Pollack, J. B. 1991, ApJ, 375, 740

Schreyer, K., Semenov, D., Henning, T., \& Forbrich, J. 2006, ApJ, 637, L129

Semenov, D., Henning, T., Helling, C., Ilgner, M., \& Sedlmayr, E. 2003, A\&A, 410, 611

Shakura, N. I. \& Sunyaev, R. A. 1976, MNRAS, 175,613

Shakura, N. I. \& Syunyaev, R. A. 1973, A\&A, 24, 337

Shepherd, D. S., Claussen, M. J., \& Kurtz, S. E. 2001, Science, 292, 1513

Stahler, S. W. \& Palla, F. 2005, The Formation of Stars (The Formation of Stars, by Steven W. Stahler, Francesco Palla, pp. 865. ISBN 3527-40559-3. Wiley-VCH , January 2005.)

Stepinski, T. F. 1998, Icarus, 132, 100

Stepinski, T. F., Reyes-Ruiz, M., \& Vanhala, H. A. T. 1993, Icarus, 106, 77

Toomre, A. 1964, ApJ, 139, 1217

Turner, N. J., Quataert, E., \& Yorke, H. W. 2007, ApJ, 662, 1052

Whitney, B. A., Wood, K., Bjorkman, J. E., \& Cohen, M. 2003, ApJ, 598, 1079
Wolf, S., Schegerer, A., Beuther, H., Padgett, D. L., \& Stapelfeldt, K. R. 2008, ApJ, 674, L101

Wolfire, M. G. \& Cassinelli, J. P. 1987, ApJ, 319, 850

Yorke, H. W. 2004, in IAU Symposium, Vol. 221, Star Formation at High Angular Resolution, ed. M. G. Burton, R. Jayawardhana, \& T. L. Bourke, 141

Yorke, H. W. \& Bodenheimer, P. 1999, ApJ, 525, 330

Zhang, Q. 2005, in IAU Symposium, Vol. 227, Massive Star Birth: A Crossroads of Astrophysics, ed. R. Cesaroni, M. Felli, E. Churchwell, \& M. Walmsley, 135-144

Zinnecker, H. \& Yorke, H. W. 2007, ARA\&A, 45, 481

This 2-column preprint was prepared with the AAS LATEX macros v5.2. 